\title{
Potencial dendroclimático de Nothofagus betuloides en la Cordillera de Darwin, Tierra del Fuego, Chile
}

\author{
A dendroclimatic analysis of Nothofagus betuloides forests from Cordillera Darwin, \\ Tierra del Fuego, Chile
}

\author{
Pamela Soto-Rogel*, Juan Carlos Aravena \\ *Autor de correspondencia: Universidad de Magallanes, Punta Arenas, Chile, \\ tel.: 56-61-2209330, pamela.soto.rogel@gmail.com
}

\begin{abstract}
SUMMARY
The lack of long and complete instrumental records hampers the study of climatic variability in remote regions of the Earth. Alternatively, paleoenvironmental sources of information such as tree rings provide valuable information on climate variations. In the context of this study, four new tree-ring width chronologies were developed from Nothofagus betuloides in Cordillera Darwin, southern Chile. The main goal of this study is to estimate the influence of the Antarctic Oscillation (AAO) and changes in temperature and rainfall on Nothofagus betuloides tree growth. New tree-ring chronologies were developed from samples collected at Glaciar

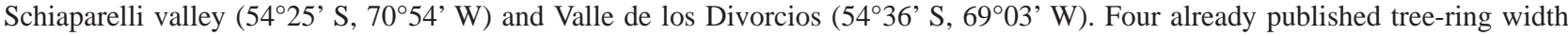
chronologies from Nothofagus betuloides in the region were also included in the analysis. Using Principal Component Analysis (PCA) on the eight tree-ring width chronologies, two main growth patterns were identified: PC1 or eastern tree-growth mode, and PC2 or western tree-growth mode. Both components showed significant correlations with air temperature and AAO. PC1 presented significant negative correlations with summer rainfall, temperature and AAO index. On the contrary, PC2 showed significant positive correlations with air temperatures, rainfall and AAO index. Significant growth responses to climate variability indicate that $N$. betuliodes records could be considered a reliable proxy of environmental fluctuations during the last few centuries in Cordillera Darwin, Chile.
\end{abstract}

Key words: Nothofagus betuloides, rainfall, temperature, AAO, Cordillera de Darwin.

\section{RESUMEN}

El estudio de la variabilidad climática en regiones remotas se dificulta por la escasez de series instrumentales largas y completas. Una alternativa es recurrir al uso de fuentes de información paleoambiental, tales como las series de anillos de crecimiento arbóreo. En este trabajo se presentan cuatro nuevas cronologías de ancho de anillos de Nothofagus betuloides para la Cordillera de Darwin. El objetivo principal fue estimar la influencia de las variaciones en temperatura, precipitación y la Oscilación Antártica (AAO) en el crecimiento radial de esta especie. Las nuevas cronologías fueron desarrolladas desde muestras colectadas en los valles Schiaparelli (54²5' S, 7054' O) y De los Divorcios (54³6' S, 6903' O). Además, se utilizaron las series de crecimiento de otras cuatro cronologías publicadas previamente. Mediante análisis de componentes principales se determinaron dos modos dominantes de variación en el crecimiento: CP1, modo de crecimiento de las cronologías ubicadas en el sector oriental de Cordillera de Darwin, y CP2, modo de crecimiento de las cronologías ubicadas en el sector occidental de la misma. Ambos componentes mostraron correlaciones significativas con la temperatura, precipitación y AAO. CP1 presentó correlaciones negativas significativas para los meses de verano con la precipitación, temperatura y AAO. En cambio, CP2 mostró correlaciones positivas significativas con las mismas variables. La significativa sensibilidad a las variaciones climáticas del crecimiento de $N$. betuloides indica que las series de ancho de anillos de esta especie pueden ser utilizadas para estimar fluctuaciones ambientales de los últimos siglos en Cordillera de Darwin.

Palabras clave: Nothofagus betuloides, precipitación, temperatura, AAO, Cordillera de Darwin.

\section{INTRODUCCIÓN}

El estudio de los cambios climáticos a nivel regional requiere de bases de datos meteorológicos con una cobertura geográfica adecuada y con la mayor longevidad posible. En el extremo sur de América del Sur se cuenta con registros escasos y de períodos breves, lo que dificulta el análisis completo de la variabilidad climática de la región. Varios autores, sin embargo, han realizado estudios regionales a pesar de esta incompleta base de datos. Villalba et al. (2003) estudiando estaciones meteorológicas entre los $37^{\circ}$ y $55^{\circ} \mathrm{S}$ en Chile y Argentina, encontraron una tendencia al calentamiento que es más marcada en la estación de verano para el sur de la Patagonia, con tendencias 
que van desde 0,15 a $0,25^{\circ} \mathrm{C}$ por década para el período 1930-1990. Quintana y Aceituno (2012), en tanto, analizan registros meteorológicos de Chile desde los $29,9^{\circ}$ a $53^{\circ}$ $\mathrm{S}$, vinculando cambios y fluctuaciones de precipitaciones con anomalías de circulación atmosférica regional. Según estos autores, en las últimas décadas, existe una tendencia negativa de las precipitaciones entre los $37^{\circ}$ y $43^{\circ} \mathrm{S}$, los que se relacionan con el patrón de circulación extratropical predominante del Hemisferio Sur: la Oscilación Antártica (AAO) o Modo Anular del Sur (SAM). El Índice AAO se define como el primer componente principal de los campos de presiones atmosféricas al sur de los $20^{\circ} \mathrm{S}$ (Thompson y Wallace 2000), y oscila entre fases positivas y negativas. Durante la fase positiva AAO, como la predominante en los últimos 50 años, se presentan anomalías de presión negativas en altas latitudes ( $\left.65^{\circ} \mathrm{S}\right)$, y anomalías de presión positivas en latitudes medias ( $\sim 45^{\circ} \mathrm{S}$ ). Estos cambios están asociados a la intensificación del anticiclón del Pacífico Sur Oriental, y por ello, con una disminución en la precipitación en latitudes medias; lo inverso se observa para la fase negativa de este mismo índice (Garreaud et al. 2009).

Una manera de paliar la escasez de datos climáticos instrumentales recién aludida, es usar registros ambientales de origen biológico a modo de proxies climáticos de alta resolución. Uno de estos proxies son las series de ancho de anillos del crecimiento radial de especies arbóreas. Villalba et al. (2012) para la región del sur de América del Sur entre los $32^{\circ}$ y $56^{\circ} \mathrm{S}$ desarrollaron cronologías de anillos de árboles de diferentes especies y compararon sus patrones de crecimiento con especies arbóreas de Nueva Zelandia y Tasmania. En el sector sur occidental de América del Sur, en la banda de los $32^{\circ}$ y $44^{\circ} \mathrm{S}$, se analizaron las especies Austrocedrus chilensis D. Don (ciprés de la cordillera) y Araucaria araucana K. Koch (araucaria, pehuén); y en la banda de los $53^{\circ}$ a los $56^{\circ} \mathrm{S}$ la especie Nothofagus betuloides Oerst (coigüe de Magallanes). En Nueva Zelandia se estudió la especie Halocarpus biformis Hook (pino amarillo, pino rosa), mientras en Tasmania las especies consideradas fueron Lagarostrobos franklinii Hook (pino Huon, pino Macquarie) y Phyllocladus aspleniifolius Labill (pino copa de apio). Las tres especies sudamericanas estudiadas muestran una clara disminución en el ancho de los anillos después de 1950, mientras que las especies de Nueva Zelandia y Tasmania presentan el patrón contrario. Las especies A. chilensis y A. araucana muestran correlación positiva significativa entre la reducción de ancho de los anillos y la disponibilidad de agua en verano; en cambio, para la especie $N$. betuloides su crecimiento está limitado por la temperatura, mostrando una correlación negativa significativa entre la temperatura y el crecimiento radial. En cuanto a la relación con el índice de Oscilación Antártica (AAO), las especies estudiadas en América del Sur presentan correlaciones negativas significativas entre el crecimiento radial y la fase positiva de este índice. En Nueva Zelandia y Tasmania, por tratarse de especies subalpinas, presentan por el contrario relaciones positivas significativas entre el crecimiento radial con las temperaturas regionales y el índice AAO.

El objetivo principal de este trabajo es estudiar la influencia de la variabilidad climática regional representada aquí por la temperatura del aire, la precipitación y las variaciones en el índice de Oscilación Antártica (AAO), en el crecimiento radial de los bosques de $N$. betuloides de la Cordillera de Darwin, Tierra del Fuego, Chile. Como objetivos específicos este estudio propone: 1) Desarrollar nuevas cronologías de ancho de anillos de crecimiento para bosques de $N$. betuloides de la Cordillera de Darwin, y comparar sus patrones de crecimiento en el contexto de cronologías previamente desarrolladas para la misma especie en el extremo sur de América del Sur. 2) Estudiar variabilidad climática mediante el análisis de datos de las diferentes estaciones meteorológicas del Sur de América del Sur e índices de Oscilación Antártica (AAO), de modo de analizar la respuesta al clima (temperatura del aire, precipitación, AAO) de las cronologías de ancho de anillos de árboles de $N$. betuloides de la Cordillera de Darwin y zonas aledañas.

La hipótesis que se pone a prueba es que las variables climáticas mencionadas han influido significativamente en los patrones de crecimiento de anchos de anillos de árboles de $N$. betuloides en las últimas décadas en Cordillera de Darwin, de modo tal que las variaciones en los patrones de crecimiento de la referida especie son un buen proxy de los cambios ambientales de la región.

\section{MÉTODOS}

Área de estudio. La Cordillera de Darwin, Tierra del Fuego $\left(54^{\circ} 45^{\prime} \mathrm{S} 69^{\circ} 29^{\prime} \mathrm{O}\right)$, recibe el efecto de frentes de actividad ciclónica polar, teniendo como consecuencia un alto porcentaje de nubes y elevada precipitación (Carrasco et al. 2002). Presenta un gradiente de precipitaciones OesteEste, con el sector oriental mostrando características más continentales; en cambio, en el sector occidental se observan condiciones húmedas homogéneas a lo largo de todo el año. Para toda esta región se reconocen tres unidades de vegetación en un gradiente altitudinal según Pisano (1977): a) Bosque Perennifolios ( $N$. betuloides y Drimys winteri J. R. Forst. y G. Forst (canelo)), b) Bosques Perennifolios Puros de $N$. betuloides, y c) Tundra Magallánica.

Desarrollo de las cronologías de anillos de crecimiento arbóreo. Las cronologías de anillos de crecimiento de $N$. betuloides se desarrollaron a partir de muestras colectadas en dos zonas en la Cordillera de Darwin, cada una con un par de sitios de colecta (cuadro 1, figura 1). Dos de los sitios están ubicados en el extremo Noroccidental de la Cordillera de Darwin, en el valle del glaciar Schiaparelli, denominados Schiaparelli 2013 (SCH-13) y Schiaparelli 2014 (SCH-14). Otros dos sitios están ubicados en el extremo suroriental de la misma cordillera, en las cercanías del valle del glaciar Dalla Vedova, siendo denominados 
Cuadro 1. Sitios de colecta y sus principales características.

Main characteristics of study sites.

\begin{tabular}{|c|c|c|c|c|c|c|c|c|}
\hline Sitio & Código & $\begin{array}{l}\text { Latitud/ } \\
\text { Longitud }\end{array}$ & $\begin{array}{l}\text { Altitud } \\
\text { (m s.n.m.) }\end{array}$ & Período & $\begin{array}{l}\text { Número } \\
\text { de radios }\end{array}$ & $\begin{array}{l}\text { Número. } \\
\text { de Árboles }\end{array}$ & $\begin{array}{c}\text { Número de } \\
\text { muestras cofechada }\end{array}$ & Fuente \\
\hline Schiaparelli 2013 & SCH-13 & $\begin{array}{l}54^{\circ} 25^{\prime} \mathrm{S} / \\
70^{\circ} 54^{\prime} \mathrm{O}\end{array}$ & 300 & $1701-2012$ & 40 & 20 & 32 & Este estudio \\
\hline Schiaparelli 2014 & SCH-14 & $\begin{array}{l}54^{\circ} 23^{\prime} \mathrm{S} / \\
70^{\circ} 52^{\prime} \mathrm{O}\end{array}$ & 300 & $1869-2013$ & 50 & 25 & 22 & Este estudio \\
\hline $\begin{array}{l}\text { Valle de Los } \\
\text { Divorcios } 2013\end{array}$ & VD-13 & $\begin{array}{l}54^{\circ} 36^{\prime} \mathrm{S} / \\
69^{\circ} 03^{\prime} \mathrm{O}\end{array}$ & 270 & $1660-2012$ & 40 & 20 & 34 & Este estudio \\
\hline $\begin{array}{l}\text { Paso de La } \\
\text { Muerte } 2014\end{array}$ & PM-14 & $\begin{array}{l}54^{\circ} 36^{\prime} \mathrm{S} / \\
6903^{\prime} \mathrm{O}\end{array}$ & 350 & $1758-2013$ & 40 & 20 & 30 & Este estudio \\
\hline Lago Despreciado & LDP & $\begin{array}{l}54^{\circ} 20^{\prime} \mathrm{S} / \\
68^{\circ} 49^{\prime} \mathrm{O}\end{array}$ & 401 & $1624-2005$ & 66 & 55 & 28 & $\begin{array}{l}\text { Villalba et } \\
\text { al. } 2012\end{array}$ \\
\hline $\begin{array}{l}\text { Lago Deseado } \\
\text { Norte }\end{array}$ & LDN & $\begin{array}{l}54^{\circ} 22^{\prime} \mathrm{S} / \\
68^{\circ} 46^{\prime} \mathrm{O}\end{array}$ & 455 & $1527-2005$ & 53 & 25 & 53 & $\begin{array}{l}\text { Villalba et } \\
\text { al. } 2012\end{array}$ \\
\hline Lago Fagnano & LFA & $\begin{array}{l}54^{\circ} 28^{\prime} \mathrm{S} / \\
68^{\circ} 41^{\prime} \mathrm{O}\end{array}$ & 310 & $1577-2005$ & 59 & 25 & 58 & $\begin{array}{l}\text { Villalba et } \\
\text { al. } 2012\end{array}$ \\
\hline Lago Róbalo & LRB & $\begin{array}{l}54^{\circ} 58^{\prime} \mathrm{S} / \\
67^{\circ} 41^{\prime} \mathrm{O}\end{array}$ & 305 & $1489-2008$ & 124 & 76 & 98 & $\begin{array}{l}\text { Llancabure } \\
2011\end{array}$ \\
\hline
\end{tabular}

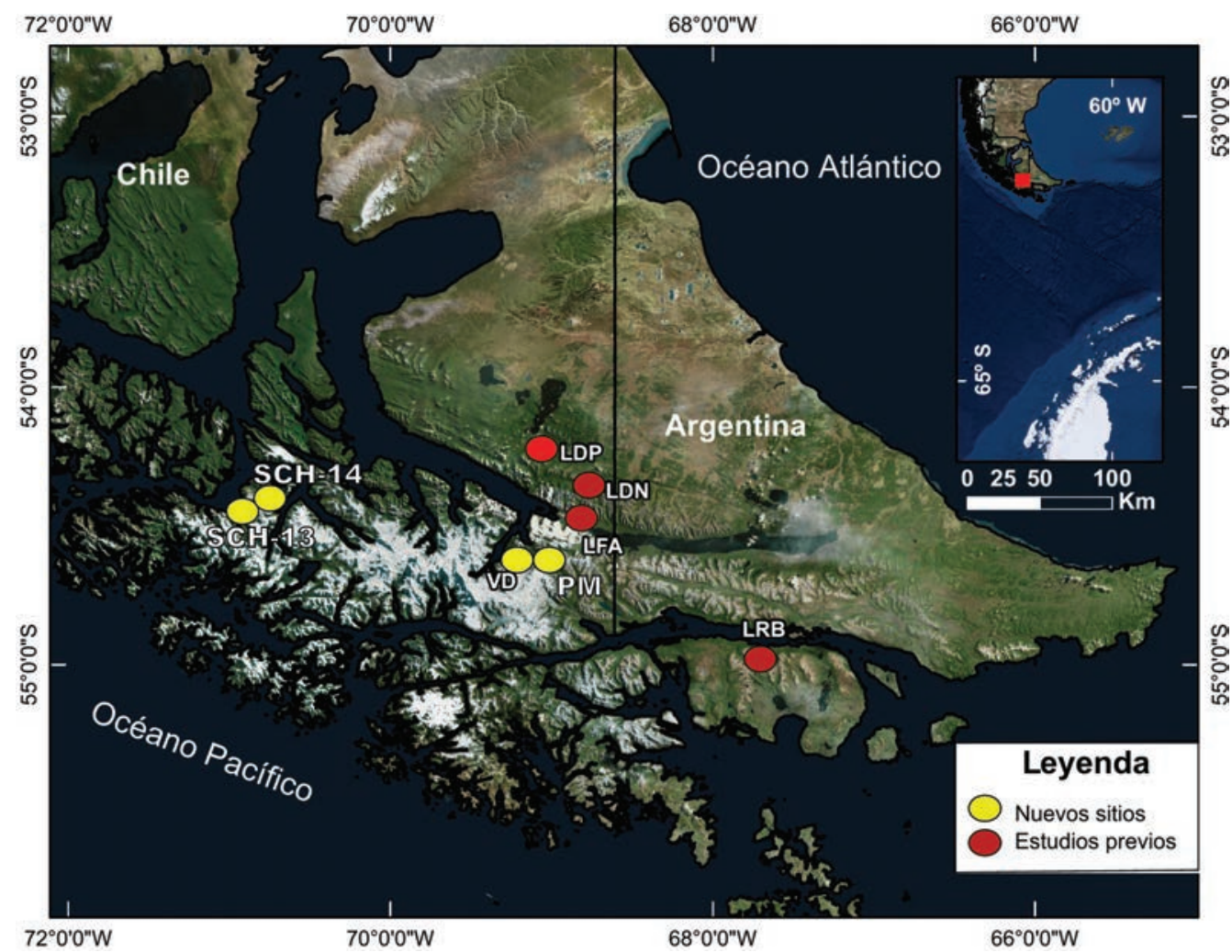

Figura 1. Mapa del área de estudio, indicando la ubicación de los sitios de colecta identificados por códigos de tres letras. Los nombres de los sitios están dados en el cuadro 1. Mapa elaborado en Quantum GIS.

Map of the study area, indicating study sites identified by three-letter codes (for details see table 1). Map made using the software Quantum GIS (Quantum GIS Development Team 2013). 
valle de los Divorcios 2013 (VD-13), y Paso de la Muerte (PM-14). Además, se analizaron las series de crecimiento de otros cuatro sitios previamente estudiados e incluidos en Villalba et al. (2012). Tres de estos sitios están ubicados de norte a sur en las proximidades del Lago Fagnano, en la porción Suroriental de Tierra del Fuego: Lago Despreciado (LDP), Lago Deseado Norte (LDN) y Lago Fagnano (LFA). El cuarto sitio de muestreo corresponde a la cuenca que alberga el Lago Róbalo (LRB) cercano a Puerto Williams, Isla Navarino. Este sitio fue desarrollado por Llancabure (2011) y también incorporado en los análisis de Villalba et al. (2012). Los sitios de estudio corresponden a bosques dominados por $N$. betuloides, con árboles de una altura promedio de $15 \mathrm{~m}$, y creciendo en sitios de elevaciones por sobre los $270 \mathrm{~m}$ s.n.m. (cuadro 1).

Las muestras de crecimiento arbóreo se prepararon siguiendo las técnicas dendrocronológicas descritas por Stokes y Smiley (1968). Luego se procedió con el fechado visual de cada muestra bajo lupa estereoscópica con un aumento de 15X. Para muestras provenientes de árboles vivos se asignó a cada anillo de crecimiento el año calendario en el cual comenzó su crecimiento. Luego de terminado el fechado visual, se procedió a la medición de los anillos de cada muestra utilizando un dendrómetro Velmex, con una precisión de $0,01 \mathrm{~mm}$. Se utilizó el programa computacional COFECHA (Holmes 1992) para evaluar la calidad de las mediciones y detectar posibles errores en la asignación de edades calendario a cada uno de los anillos de crecimiento. Las cronologías de anchos de anillos fueron desarrolladas a partir de las series correctamente fechadas, utilizando el paquete de la librería de programas dendrocronológicos (dplR; Bunn 2008) en el programa de libre acceso R Project.

La estandarización de las series de crecimiento se realizó ajustando cada serie a una curva exponencial negativa o a una regresión lineal. A través de este ajuste se reduce el efecto de la disminución del ancho de los anillos de crecimiento debido a la edad del árbol, o cambios en su geometría, y se preserva la varianza de baja frecuencia presente en las series. La curva resultante estandarizada presenta una media igual a $1 \mathrm{y}$ una varianza relativamente homogénea a lo largo de los años (Fritts 1976). El programa dplR entrega los resultados estadísticos de la cronología que evalúan su calidad (cuadro 3), incluyendo parámetros tales como sensibilidad media, una medida de las diferencias relativas en el ancho de anillos adyacentes, el coeficiente de correlación media (R-BAR) estima que porcentaje de la varianza es común entre las series (Fritts 1976), y la señal expresada de la población (EPS), una medida de la confianza de la señal común de las cronologías resultantes, y que se puede expresar como una proporción de la varianza común a la varianza total de una cronología teórica infinitamente replicada (Briffa 1995).

Análisis de componentes principales (ACP). Se realizó un análisis de componentes principales (ACP) de la red de cronologías de $\mathrm{N}$. betuloides definida para este estudio, para examinar similitudes y diferencias en sus patrones de crecimiento (Cook et al. 1994). El ACP identifica los patrones dominantes de la variabilidad del crecimiento y reduce el número de variables. En este estudio, la información presente en las ocho cronologías de ancho de anillos de crecimiento fue condensada en dos componentes principales significativos, CP-1 y CP-2. EL ACP fue desarrollado en el programa de libre acceso $\mathrm{R}$ project. Se usó la fórmula principal de ACP del R Project, con la matriz de datos con un número total de ocho ítems (sitios) $\mathrm{y} n$ variables ( $\mathrm{n}=$ número de años que se utilizan para el análisis), con un rango temporal de aproximadamente 90 años, determinado por el período común a todos los sitios (1917 al 2005).

Estaciones meteorológicas e índice de Oscilación Antárti$\mathrm{ca}(\mathrm{AAO})$. Se utilizaron datos de las estaciones meteorológicas a cargo de la Dirección Meteorológica de Chile (DMC), de sus informes climatológicos tanto de precipitación como de temperatura, de las estaciones Carlos Ibáñez, Punta Arenas, Guardia Marina Zañartu, Puerto Williams, y Evangelistas a cargo del Servicio Meteorológico de la Armada de Chile (SMA). En el caso de Argentina los datos climatológicos se obtuvieron de la base de datos Global Historical Climatology Network - (GHCN) (cuadro 2) a partir del Servicio Meteorológico Nacional (Argentina) (SMN).

En este trabajo además se incluyó al índice de Oscilación Antártica (AAO) como una de las variables climáticas que puede estar influenciando el crecimiento de $N$. betuloides. El índice AAO de la NOAA (National Oceanic and Atmospheric Administration) es definido como el modo de variabilidad predominante de las anomalías promedio mensuales de presión atmosférica a los 700 hPa de altura geopotencial $(\mathrm{GpH})$, al sur de los $20^{\circ} \mathrm{S}$ (Thompson y Wallace 2000). El cálculo de este índice se hace usando datos derivados de modelos meteorológicos numéricos predictivos, a partir de información satelital con un rango temporal de 1979 al 2015.

Se realizaron análisis de correlación de Pearson entre las estaciones meteorológicas indicadas en el cuadro 2 y el índice de Oscilación Antártica (AAO-NCEP). Tales correlaciones consideraron un nivel de confianza de $95 \%$ con dos grados de libertad. Para obtener las correlaciones y el nivel significancia al $95 \%$ se utilizó el paquete estadístico R, con las rutinas “corrplot” y la función “cor.mtest” (Wei 2013).

Análisis crecimiento-clima. Para estudiar la relación entre el crecimiento arbóreo y el clima regional se usaron funciones de correlación que relacionan los índices de ancho de anillos con índices mensuales de AAO y/o anomalías mensuales de temperatura y precipitación. A través del análisis de funciones de correlación se identificaron las variables climáticas y meses que presentan las relaciones más fuertes con el ancho de anillo a través del programa Rproject con el paquete estadístico "bootRes" (Zang y Bion- 
Cuadro 2. Estaciones climáticas usadas en el análisis dendroclimático. DMC: Dirección Meteorológica de Chile, SMA: Servicio Meteorológico de la Armada de Chile y SMN: Servicio Meteorológico Nacional (Argentina). Pp: Precipitación, Tmed: temperatura media, Tmin: temperatura mínima media y Tmax: temperatura máxima media.

Meteorological stations used in this study. Source: DMC: Dirección Meteorológica de Chile, SMA: Servicio Meteorológico de la Armada de Chile and SMN: Servicio Meteorológico Nacional (Argentina). Pp: precipitation, Tmed: mean temperature, Tmin: minimum temperature and Tmax: maximum temperature.

\begin{tabular}{|c|c|c|c|c|}
\hline Estación & Variable & Latitud/Longitud & Período & Fuente \\
\hline \multirow{2}{*}{ Faro Evangelista (EVA) } & \multirow{2}{*}{ Pp } & $52^{\circ} 40^{\prime} \mathrm{S}$ & \multirow{2}{*}{$1899-2000$} & \multirow{2}{*}{ SMA } \\
\hline & & $75^{\circ} 06^{\prime} \mathrm{O}$ & & \\
\hline \multirow{4}{*}{ Punta Arenas (PUQ) } & Pp & & $1900-2014$ & \multirow{4}{*}{ DMC } \\
\hline & Tmed & $53^{\circ} 9^{\prime} 10^{\prime \prime} \mathrm{S}$ & $1888-2014$ & \\
\hline & Tmin & $70^{\circ} 55^{\prime} 35^{\prime \prime} \mathrm{O}$ & $1930-2014$ & \\
\hline & Tmax & & 1930-2014 & \\
\hline \multirow{4}{*}{ Puerto Williams (PWT) } & Pp & & 1968-2014 & \multirow{4}{*}{ DMC } \\
\hline & Tmed & $54^{\circ} 55^{\prime} 54^{\prime \prime} \mathrm{S}$ & $1968-2014$ & \\
\hline & Tmin & $67^{\circ} 36^{\prime} 56^{\prime \prime} \mathrm{O}$ & $1968-2014$ & \\
\hline & Tmax & & 1968-2014 & \\
\hline \multirow{2}{*}{ Ushuaia (USH) } & $\mathrm{Tm}$ & $54^{\circ} 48^{\prime} 0^{\prime \prime} \mathrm{S}$ & $1901-2014$ & \multirow{2}{*}{ SMN } \\
\hline & $\mathrm{Pp}$ & $68^{\circ} 08^{\prime} 06^{\prime \prime} \mathrm{O}$ & 1931-2014 & \\
\hline
\end{tabular}

di 2013). Las correlaciones entre las series de anillos y clima regional se efectuaron para un período de 16 meses, a partir de enero de la estación de crecimiento anterior a abril del año siguiente al inicio de la formación del anillo. Se considera el año anterior en el análisis puesto que el ancho de los anillos resulta de la integración de los procesos de influencia climática ocurridos sobre un extenso período que puede llegar a abarcar el año previo (Fritts 1976).

Como una forma de extender el análisis de la relación entre el crecimiento arbóreo y el clima a una expresión de la variabilidad climática en un ámbito geográfico más amplio, se usaron también funciones de correlación entre el crecimiento arbóreo de $N$. betuloides y las variaciones de deuterio $\left(d^{2} \mathrm{H}\right.$, un proxy de las variaciones de la temperatura del aire), en un testigo de hielo obtenido en la isla James Ross en el sector nor-oriental de la Península Antártica (Abram et al. 2013). Además, se analizó la relación entre el mismo crecimiento arbóreo y la reconstrucción del AAO conseguida a partir de las estimaciones de temperatura basados en el mismo testigo de hielo y otros dos proxies sensibles a la temperatura (Abram et al. 2014).

Análisis de series de tiempo. Para determinar las frecuencias dominantes en el crecimiento de $N$. betuloides, representado por los Componentes Principales CP-1 y CP2, se utilizó el método espectral multi-taper mediante el software R a través del paquete estadístico " $d p l R$ ” (Bunn 2008). Otros análisis de las series de tiempo utilizadas en este estudio incluyeron las transformaciones wavelet y cross-wavelet. En el primer caso se buscó determinar qué tan estables y significativas en el tiempo son las frecuencias dominantes identificadas con el método espectral multi-taper recién descrito para las series de CP-1 y CP-2. De esta forma se busca indagar la presencia de modos característicos en la periodicidad de las series analizadas de manera de identificar posibles forzantes climáticos asociados a los patrones de crecimiento. Finalmente, el análisis de cross-wavelet permitió explorar una posible concordancia en la estabilidad y significancia de las frecuencias características de CP-1 y CP-2 con aquellas descritas para el índice de Oscilación Antártica AAO-NCEP, de forma de indagar en señales espectrales de alta potencia comunes entre ambas variables. Para los análisis de wavelet y crosswavelet se usó el paquete estadístico "biwavelet" (Gouhier et al. 2016).

\section{RESULTADOS}

Variabilidad climática regional. Las temperaturas medias del aire de la estación meteorológica de Punta Arenas estuvieron altamente relacionadas con todas las otras estaciones consideradas, con valores de correlación por encima de 0,69. La correlación varió dependiendo de la combinación de meses (estaciones del año) que se consideró, con los valores más altos para los meses de verano. Las relaciones más débiles en temperaturas medias se dieron entre las estaciones meteorológicas de Ushuaia y Río Gallegos para todas las comparaciones efectuadas, en especial en el caso de las temperaturas medias anuales. De todos modos, para el caso de esta variable climática se cumplió la ca- 
racterística regional con valores de correlación altos en su mayoría por sobre 0,5. Esto comprobó que la temperatura es una variable climática de expresión regional uniforme con variaciones graduales en escalas espaciales amplias.

En cuanto a las relaciones entre precipitaciones de las diferentes estaciones meteorológicas, estas resultaron mayoritariamente no significativas, con excepción de las comparaciones para la precipitación anual de las estaciones de Evangelista y Punta Arenas, la precipitación de otoño y primavera de las estaciones de Punta Arenas y Río Gallegos, y la precipitación de verano de las estaciones de Punta Arenas y Ushuaia. Este resultado confirmó la naturaleza más heterogénea de la distribución espacial de las precipitaciones, expresándose en fuertes gradientes de esta variable climática determinados por barreras topográficas (cadenas montañosas), distancia respecto del origen de las tormentas más frecuentes, cambios en la circulación atmosférica regional, entre otras causas.

Los valores de coeficiente de correlación entre los índices de Oscilación Antártica y las variables climáticas temperatura y precipitación de diferentes estaciones meteorológicas mostró claras diferencias. Para el caso de la temperatura del aire los valores más altos de correlación se obtuvieron entre el índice AAO-NCEP con la temperatura máxima de Punta Arenas, resultando significativo para casi todos los meses del año, a excepción de agosto y septiembre. En el caso de las precipitaciones los valores de correlación no mostraron una relación significativa con AAO-NCEP y las precipitaciones de Punta Arenas, solo resultando tres meses (enero, octubre y diciembre) al borde de la significancia.

Cronologías de ancho de anillos. Se desarrollaron ocho cronologías a través del paquete estadístico dplR (figura 2), evaluando varias de sus características principales usando las versiones residuales de las cronologías (cuadro 3).

Las dos cronologías desarrolladas para el sector de Schiaparelli (Monte Sarmiento), sector oeste de la Cordillera de Darwin, presentaron similitudes a partir de 1950 con valores mayoritariamente sobre la media a excepción de $\mathrm{SCH}-13$ que para los últimos años presentó una tendencia negativa abrupta (figura 2). En el caso de las dos cronologías del sector oriental de la Cordillera de Darwin, VD-13 y PM-14, coincidieron en depresiones de crecimiento centradas alrededor de los años 1840 y 2000 . En el caso de la cronología de PM-14 comenzó esta depresión en 1985, mientras en VD-13 esta misma disminución en el crecimiento comenzó en 1990. Excepcionalmente PM-14 presentó valores por sobre la media para 1920, no mostrándose este patrón para VD-13. De este modo, las cuatro nuevas cronologías desarrolladas en este trabajo presentaron baja tasa de crecimiento en los años más recientes, aunque existen diferencias en las cuantías de las mismas.

De los cuatros cronologías previamente utilizadas en Villalba et al. (2012), tres fueron desarrolladas en localidades muy cercanas: Lago Despreciado (LDP), Lago Deseado Norte (LDN) y Lago Fagnano (LFA). Estas tres cronologías presentan similitudes en el modo de variabilidad, en cuanto a valores sobre la media entre 1800-1850 y para el período 1980-1990, y mostraron como años marcadores 1769-1770 con valores de crecimiento muy por debajo de la media, además presentaron una marcada disminución de crecimiento para los últimos años de las cronologías (figura 2).

La cuarta cronología previamente publicada es Lago Róbalo (LRB) en Isla Navarino presentó valores mayoritariamente por sobre la media entre 1780-1820, pero además se observaron al menos cuatro depresiones notables con valores por bajo la media (1613, 1647-1687, 1705-1744 y 1985-2008). La última depresión entre 1995-2008, fue la depresión más abrupta mostrada en este trabajo, muy similar a $\mathrm{SCH}-13$ (figura 2).

Patrones de crecimiento arbóreo. Basados en las cronologías residuales de aproximadamente 100 años, los tres prim-eros componentes principales explicaron en conjunto más del $77 \%$ de la varianza total (figura $3 \mathrm{~A}$ ). El CP-1 explicó el 53,5\% de la varianza total del crecimiento de los anillos de árboles (figura 3A). Los valores de carga mayores a 0,5 estuvieron dados para los sitios de Lago Despreciado, Lago Deseado Norte, Lago Fagnano, Lago Róbalo,

Cuadro 3. Estadísticos descriptivos para las cronologías de Nothofagus betuloides (versiones estándar, códigos según cuadro 1).

Statistics for $N$. betuloides residual chronologies (for site details see table 1).

\begin{tabular}{lcccccccc}
\hline \multirow{2}{*}{ Estadísticos descriptivos } & \multicolumn{7}{c}{ Cronologías de ancho de anillo } \\
\cline { 2 - 8 } & SCH-13 & SCH-14 & VD-13 & PM-14 & LDP & LDN & LFA & LRB \\
\hline Desviación estándar & 0,401 & 0,363 & 0,282 & 0,337 & 0,313 & 0,387 & 0,414 & 0,391 \\
Ancho promedio (mm) & 0,980 & 1,090 & 0,750 & 0,820 & 1,120 & 0,830 & 0,900 & 0,890 \\
Autocorrelación & 0,782 & 0,703 & 0,817 & 0,764 & 0,737 & 0,814 & 0.824 & 0,803 \\
Sensibilidad media & 0,194 & 0,183 & 0,174 & 0,207 & 0,211 & 0,210 & 0,209 & 0,206 \\
EPS (> 0,85) & 1810 & 1920 & 1780 & 1810 & 1745 & 1630 & 1655 & 1540 \\
R-BAR & 1810 & 1920 & 1780 & 1810 & 1795 & 1680 & 1730 & 1640 \\
\hline
\end{tabular}



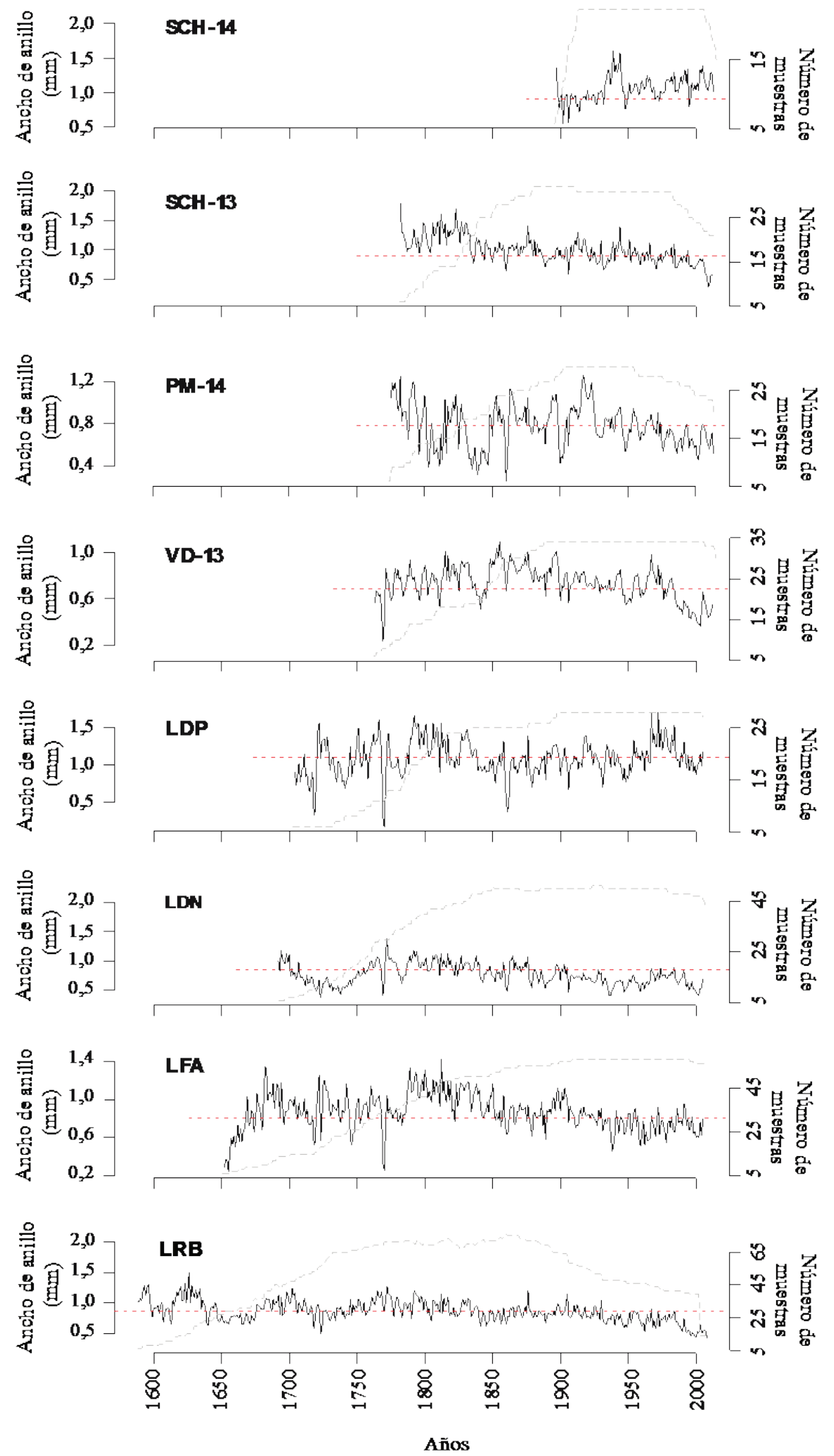

Figura 2. Cronologías de ancho de anillos $(\mathrm{mm})$ de la especie $\mathrm{N}$. betuloides (línea negra), número de muestras por año a partir de $\mathrm{n}>$ 5 (línea gris segmentada), y media de ancho de anillos (línea roja segmentada). Códigos según cuadro1. Cronologías desarrolladas a través del paquete estadístico dplR en el programa R Project (Bunn 2008).

Tree-ring width chronologies (black line) and sample depth (gray). Chronologies were developed using the Dendrochronology Program Library in R (dplR) (Bunn 2008). 

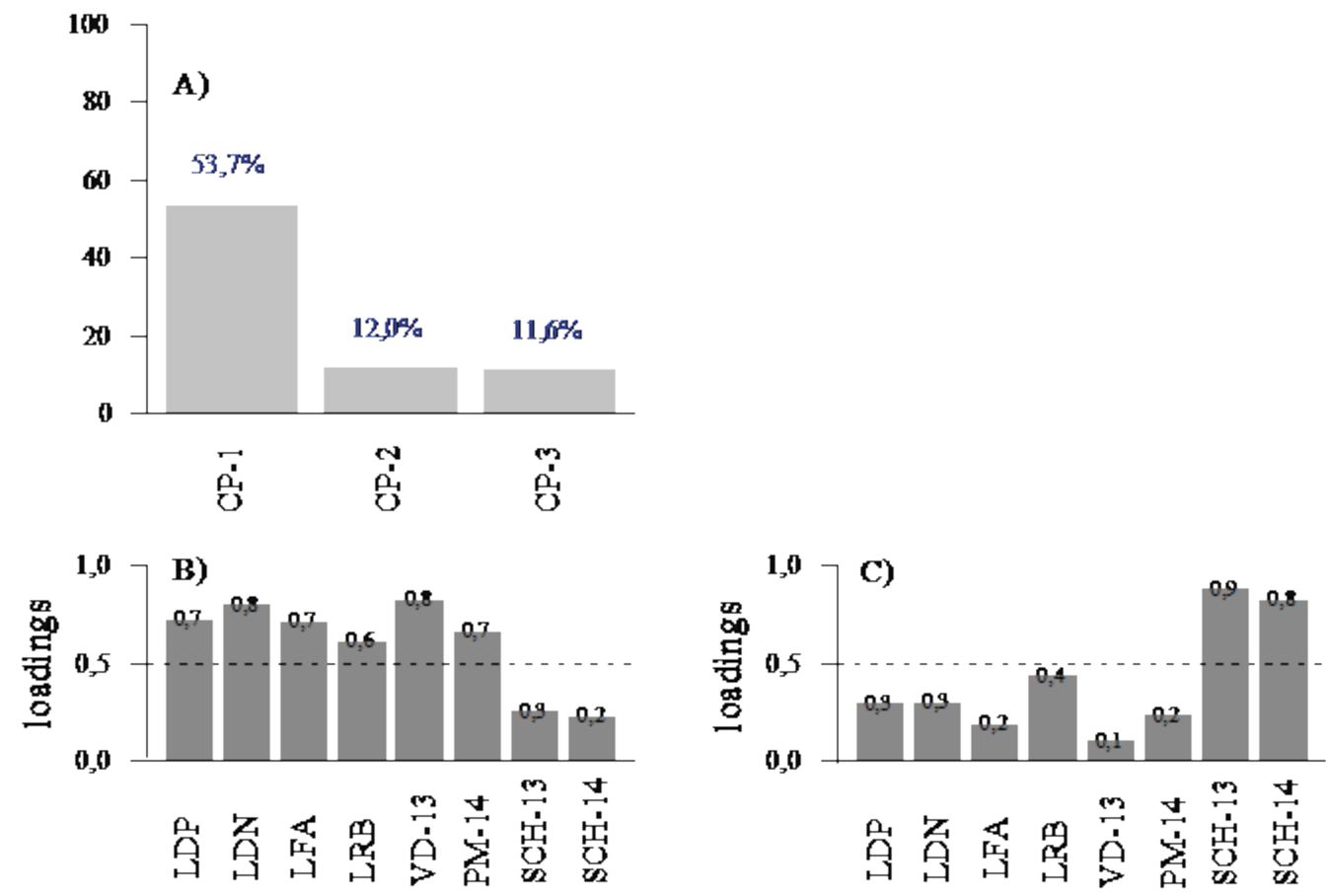

Figura 3. Análisis de componentes principales (ACP) de las cronologías residuales de $N$. betuloides de los diferentes sitios de estudio para el período en común 1917-2005. A) Varianza explicada por cada componente principal, B) y C) carga (o peso) que tiene cada sitio en el CP-1 y en el CP-2, respectivamente. ACP desarrollado en R Project.

Principal Component Analyses (PCA) of the N. betuloides residual chronologies over the period 1917-2005 (site codes in table 1). A) Explained variance by each principal component; B) and C) loadings of each site in PC-1 and PC-2, respectively. PCA analyses based on R project software.

Valle de Los Divorcios y Paso de La Muerte. Los sitios con menor carga o representación en este modo de variación fueron Schiaparelli 2013 y Schiaparelli 2014 (figura 3B). Los valores de carga de los sitios correspondiente al sector oriental de Tierra del Fuego estuvieron todos por sobre 0,5. El segundo componente principal explicó $12 \%$ de la varianza total del crecimiento de los anillos de árboles (figura 3A). Los valores de carga mayores a 0,5 estuvieron dados para los sitios de Schiaparelli 2013, Schiaparelli 2014 (figura 3C). Los registros provenientes de los sitios restantes tuvieron cargas por debajo de los 0,5.

La serie de tiempo del CP-1 basado en las cronologías residuales (figura 4A) no presentó una tendencia clara en el período analizado, con valores que fluctuaron en torno al promedio entre 1917 y 1965, con un leve sesgo hacia valores negativos (ver línea de tendencia roja en la figura 4A). En la segunda mitad de la década de los 1960s la serie del CP-1 alcanzó los valores máximos de todo el período, iniciándose a partir de esa fase una disminución persistente hasta inicios del siglo 21, con una subida en los últimos cinco años.

La serie de tiempo asociada al CP-2 tampoco mostró una tendencia general clara, con valores que también fluctúan en torno al promedio (figura 4B). Comparada con la serie temporal del CP-1 esta serie tiene una mayor variabilidad de alta frecuencia y sus fluctuaciones tienden a estar en contrafase con las del CP-1.
Relación entre los patrones de crecimiento y el clima regional. Utilizando la información climática de la estación meteorológica de Punta Arenas se encontró una relación positiva significativa entre $\mathrm{CP}-1$ del crecimiento de N. betuloides con las temperaturas mínimas de febrero del año de crecimiento (figura 5A). Igualmente, se verificó una correlación positiva significativa entre el CP-2 del crecimiento arbóreo de $\mathrm{N}$. betuloides y las temperaturas medias de Punta Arenas para enero y febrero en curso (figura 5B).

Para la estación meteorológica de Ushuaia se encontró una relación significativa negativa entre $\mathrm{CP}-1$ del crecimiento arbóreo de $N$. betuloides con la precipitación del mes febrero del año de crecimiento previo (figura 5C). Por su parte, el CP-2 del crecimiento arbóreo de $N$. betuloides presentó una relación positiva y significativa con las precipitaciones para el mes de febrero del año de crecimiento previo (figura 5D). Para los valores de AAO-NCEP (figura 5E y 5F), el CP-1 del crecimiento arbóreo presentó una correlación negativa significativa para el mes de diciembre en curso, con un valor de -0,5, el más alto encontrado en este análisis. No se encontraron correlaciones significativas entre CP-2 y el índice AAO (figura 5F).

Isótopos de deuterio vs patrones de crecimiento arbóreo. El análisis de correlación de Pearson entre registros de isótopos de deuterio $\left(\mathrm{d}^{2} \mathrm{H}\right)$, obtenidos a partir de un tes- 

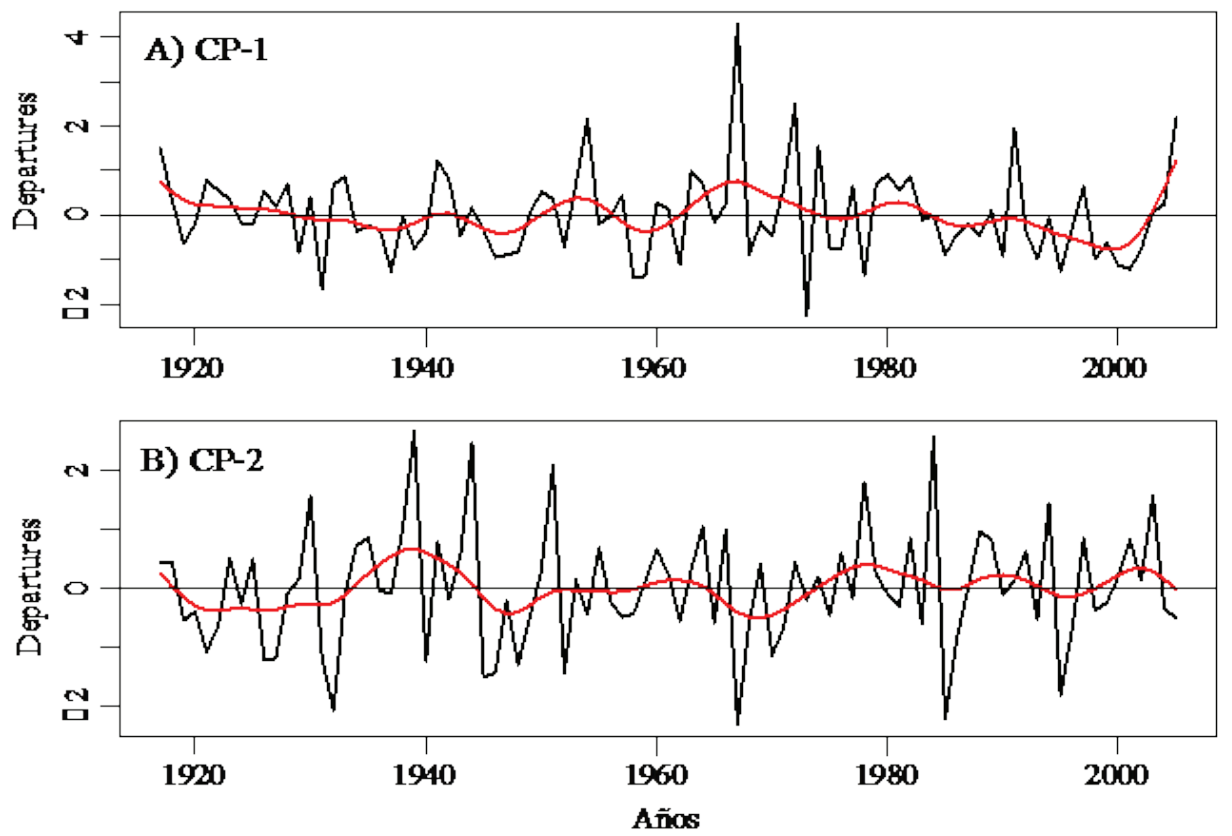

Figura 4. Series temporales de los componentes principales CP-1 (A) y CP-2 (B) en base a la matriz de correlación de las cronologías residuales de $N$. betuloides para el período en común 1917-2005 (línea gris), spline (línea roja). El ACP fue desarrollado en el contexto de R Project.

Principal component time scores of PC-1 (A) and PC-2 (B) based on the correlation matrix of the residual chronologies N. betuloides for the common period 1917-2005 (gray line). PCA analyses based on R project software.

tigo de hielo obtenido en la isla James Ross, en el sector nor-oriental de la Península Antártica (Abram et al. 2011), y los patrones de crecimiento arbóreo representados por los CP de las versiones estándar de las cronologías de ancho de anillos desarrolladas en este trabajo, mostraron que CP-1 presentó las más altas correlaciones con las anomalías de deuterio con un valor de correlación negativa de -0,56 (figura 6A). Las curvas siguen una variación inversa con un buen ajuste de sus tendencias. En el caso de la comparación con el segundo componente principal CP-2 la relación es positiva, con un valor de $\mathrm{r}=0,35$ (figura $6 \mathrm{~B}$ ). El ajuste entre ambas series se mantiene para casi todo el período de comparación, con un desacople después de aproximadamente el año 1985.

Otra comparación realizada fue entre la serie de la reconstrucción del índice AAO obtenida a partir del registro de deuterio de Abram et al. (2014) y los dos primeros componentes principales de las cronologías de anillos de crecimiento arbóreo de $N$. betuloides consideradas en este estudio (figura 6C y D). Al igual que con la serie de deuterio, CP-1 nuevamente presentó una alta correlación inversa con la serie de la reconstrucción del AAO, con un valor de -0,52 (figura 6C). El ajuste en este caso, aunque de orden inverso, es particularmente bueno a partir de aproximadamente el año 1950. La comparación entre el segundo componente principal del crecimiento arbóreo y la reconstrucción del AAO, muestra una correlación posi- tiva significativa, aunque más débil, con un valor de 0,20 (figura 6D).

Análisis de series de tiempo. Para el caso del crecimiento arbóreo de $N$. betuloides, el análisis espectral se realizó sobre los dos primeros componentes principales de la red de cronologías de esta especie (figura 7). El poder espectral del CP-1 (figura 7A) muestra una frecuencia dominante, marginalmente significativa, por sobre el límite de confianza de $90 \%$, para el período de 16 años. En el caso del CP-2 (figura 7B), el poder espectral resulta significativo para el período de 2,5 años (frecuencia $=0,4$ ), y marginalmente significativo para un período de 46 años (frecuencia $=0,022$ ).

La transformada Wavelet de CP-1 (figura 7C) mostró una persistencia para el período de dos a cinco años entre 1955 a 1980 y también alrededor de la frecuencia de los 16 años desde 1935 hasta el presente, siendo esta última no significativa. En cambio, CP-2 (figura 7D) mostró una persistencia temporal constante a través del tiempo para el período de 2,5 años.

En el caso del análisis de cross-wavelet, para CP-1 y el índice AAO-NCEP la figura 8A mostró una amplia sección de alta interrelación para el período de 2 a 4 años para el rango de años 1985-2002. Por otro lado, la figura 8B comparando CP-2 con AAO-NCEP mostró una señal espectral común entre ambas variables para el período de 3,5 años, pero solo para un muy estrecho rango de años (1995-2000). 


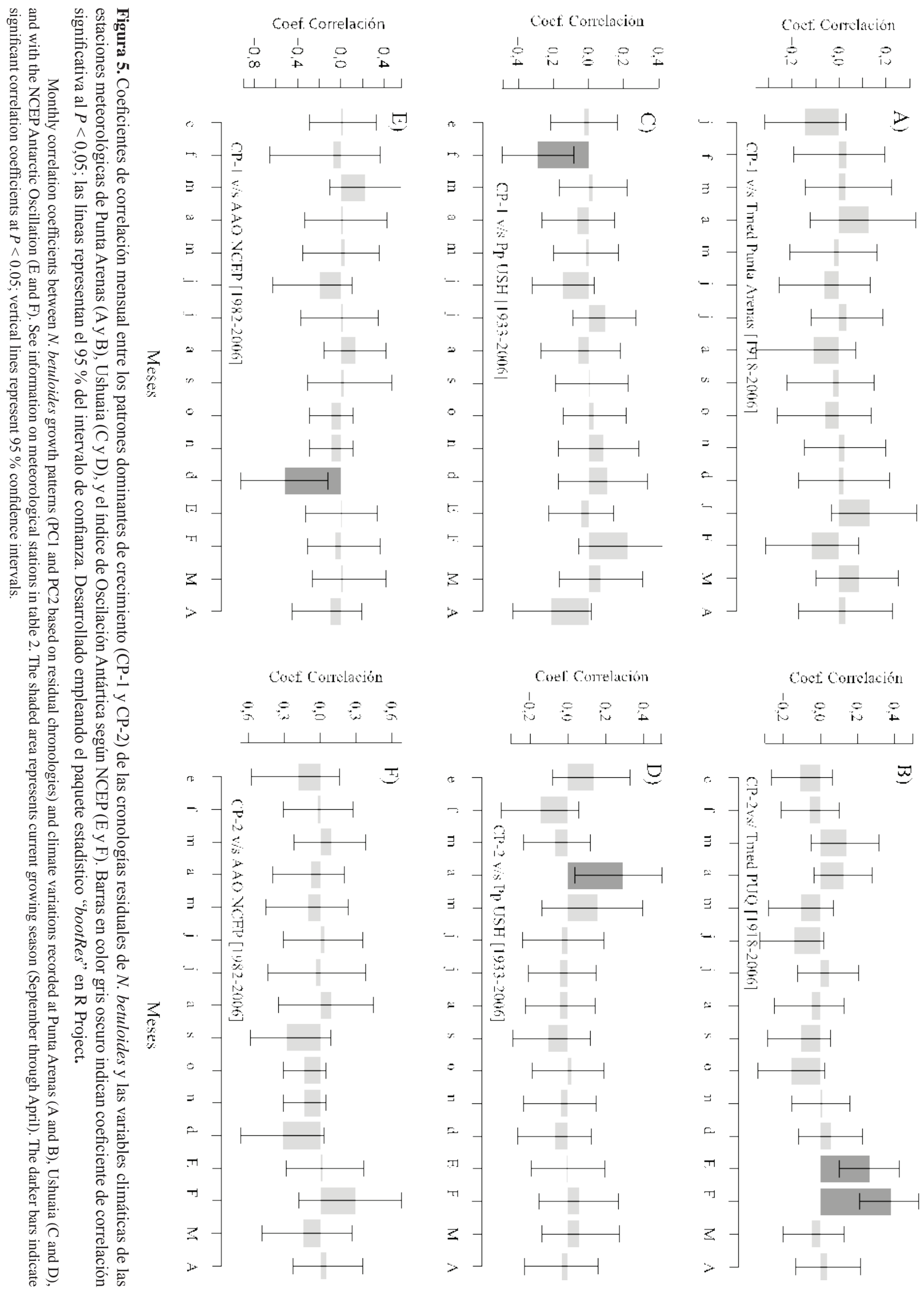



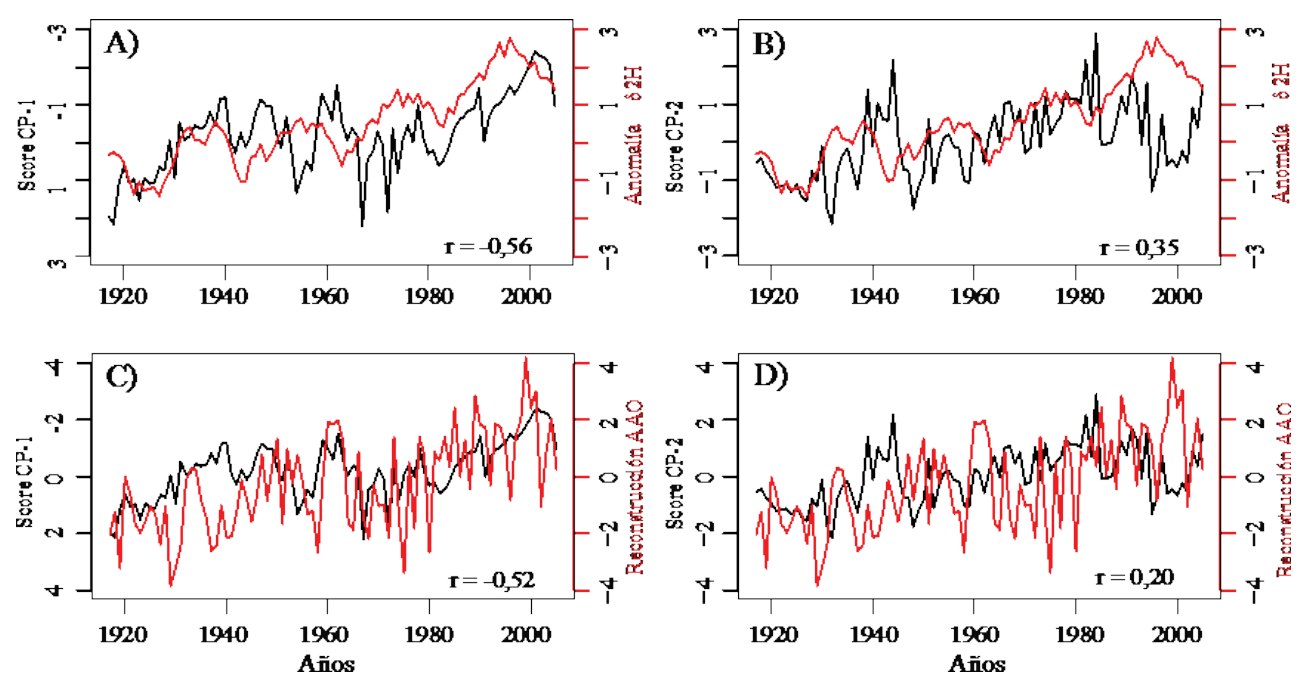

Figura 6. Relaciones entre los patrones de crecimiento arbóreo (CP-1 y CP-2) obtenidos a partir de la versión estándar de las cronologías y las anomalías de Deuterio $\left(\mathrm{d}^{2} \mathrm{H}\right)$ obtenidas desde un testigo de hielo de la Isla James Ross, Antártica (A y B), y la reconstrucción del índice AAO (C y D), obtenida a partir del mismo testigo de hielo de la Isla James Ross y otras dos reconstrucciones de las fluctuaciones de temperatura usando otros proxies paleoambientales (Abram et al. 2014). Análisis desarrollado en R Project.

Correlation analyses between growth patterns (PC1 and PC2) based on $N$. betuloides standard chronologies and Deuterium anomalies ( $\mathrm{d}^{2} \mathrm{H}$ ) recorded from an ice core in James Ross Island (A and B), and the AAO reconstruction modelled from the same ice core (C and D). Results based on R Project software.
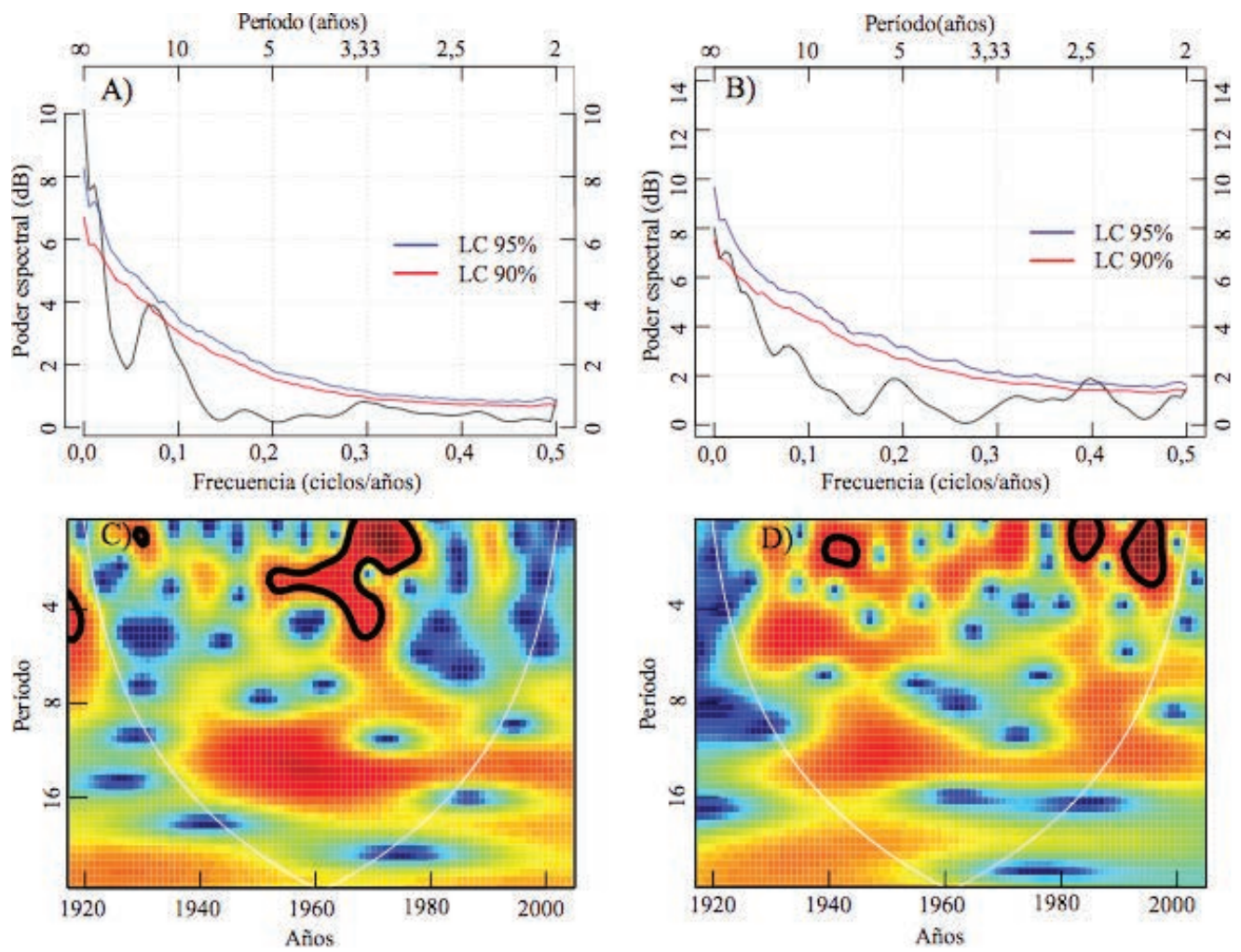

Figura 7. Poder espectral de los componentes principales de las cronologías estándar, CP-1 (A) y CP-2 (B). La línea continua roja (azul) indica $90 \%$ (95\%) del límite de confianza. Wavelet Transform para los componentes principales de las cronologías estándar, CP-1 (C) y CP-2 (D), para el período de 1917-2005. Tonos más cálidos indican una señal espectral más fuerte, mientras que los sectores con contorno negro indican significancia estadística con un límite de confianza al $95 \%$. La línea blanca delimita la zona donde el análisis es confiable.

Spectral analysis on PC-1 (A) and PC-2 (B) from the N. betuloides standard chronologies. Continuous red (blue) line indicates $90 \%$ (95\%) confidence limit. Wavelet Transform on the same principal components, PC-1 (C) and PC-2 (D), for the common period 1917-2005. Dark red indicates strong spectral power, zones enclosed by black lines indicate confidence levels above $95 \%$. White line defines the conical influence zone. 

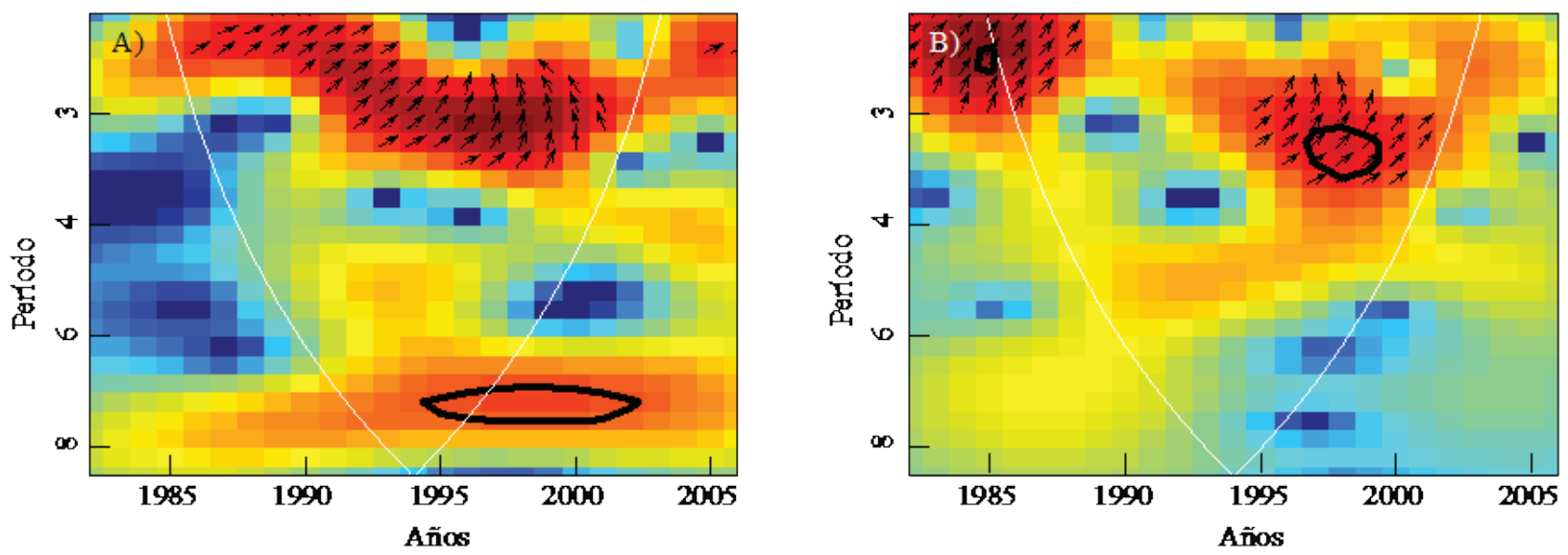

Figura 8. Análisis cross-wavelet para el período de 1982-2006 entre los componentes principales de las cronologías residual CP-1 y CP-2 y el índice AAO-NCEP (A y B, respectivamente). Tonos más cálidos indican una señal espectral más fuerte, mientras que los sectores con contorno negro indican significancia estadística con un límite de confianza al $95 \%$. La línea blanca delimita la zona donde el análisis es confiable.

Cross-wavelet analyses over the common period 1982-2006 among the principal components PC1 (A) and PC2 (B) of the N. betuloides residual chronologies and AAO-NCEP index for December. Warm colors indicate strong spectral power, zones enclosed by black lines indicate confidence levels above $95 \%$. White line defines the conical influence zone.

\section{DISCUSIÓN}

El análisis de la variabilidad climática presentado en este trabajo permite reafirmar la información previamente publicada acerca de la influencia del AAO en la distribución latitudinal de las precipitaciones y temperaturas de América del Sur y Antártica. En latitudes medias de América del Sur el AAO tiene una expresión significativa en la precipitación (Gillett et al. 2006), y variables asociadas tales como los caudales de río (Lara et al. 2008). En cambio, en el extremo sur de América del Sur y Península Antártica, este efecto se hace más evidente para el caso de las fluctuaciones de la temperatura (Gillett et al. 2006, Villalba et al. 2012, Abram et al. 2014). Este trabajo confirma la relación positiva y altamente significativa entre $\mathrm{AAO}$ y las temperaturas de la región austral de América del Sur para casi todos los meses del año.

La diferenciación geográfica Este-Oeste de los sitios de estudio que arrojó el análisis de componentes principales (ACP) tiene varias implicancias para el conocimiento de los patrones de crecimiento de los bosques de $\mathrm{N}$. betuloides en la Cordillera de Darwin y alrededores. El patrón de crecimiento del primer componente principal (CP-1), con mayor influencia de los sitios localizados hacia el Este de la Cordillera de Darwin, entre los que se incluyen las localidades ubicadas cercanas al lago Fagnano, en el sector central de Tierra del Fuego, las del Valle de Los Divorcios en el extremo oriental de la Cordillera de Darwin, y las del valle del río Róbalo en la isla Navarino, da cuenta de ambientes en términos relativos menos húmedos y sujetos a fluctuaciones térmicas más amplias durante el año, con condiciones relativamente menos húmedas y de mayor temperatura en los meses estivales. Este patrón de crecimiento Oriental refleja además una marcada fase de disminución del ancho de los anillos de crecimiento arbóreo para las últimas cinco décadas, que coincide con las tendencias hacia menor precipitación y mayor temperatura que marcan estaciones meteorológicas como la de Punta Arenas. No es de extrañar, entonces, que el crecimiento arbóreo del sector oriental de la Cordillera de Darwin responda negativamente a las elevadas temperaturas y menores precipitaciones. La misma respuesta negativa se observa cuando se comparan las tendencias de este patrón de crecimiento oriental y la fase positiva del índice de Oscilación Antártica. Estos resultados, previamente reportados por Villalba et al. (2012), pueden ser explicados por el efecto del déficit hídrico que se produciría durante el período de crecimiento, por la menor disponibilidad de agua y el aumento de las temperaturas para las últimas décadas (Llancabure 2011). De este modo, a menor humedad y temperaturas más altas durante las últimas décadas el crecimiento arbóreo de $N$. betuloides ha presentado una tendencia decreciente.

Por otro lado, el segundo componente principal (CP-2), que expresa las tendencias en el crecimiento arbóreo principalmente de las localidades del extremo occidental de la Cordillera de Darwin, en el valle del glaciar Schiaparelli, representa un patrón de crecimiento antes no descrito para los bosques de $N$. betuloides del extremo austral de Sudamérica. De este modo, este trabajo amplía el conocimiento de la variabilidad en el crecimiento de los bosques de $N$. betuloides, mostrando diferencias significativas asociadas al eje geográfico Este-Oeste en distancias no superiores 
a $220 \mathrm{~km}$, en el sistema montañoso de la Cordillera de Darwin. La respuesta al clima que presenta este patrón de crecimiento occidental es completamente opuesta al que presenta CP-1. En este caso CP-2 responde positivamente a las relativamente menores temperaturas y más elevadas precipitaciones, y a la fase positiva del índice de Oscilación Antártica. El patrón de crecimiento occidental reconocido en este trabajo, sería similar al descrito para los bosques de Nueva Zelandia y Tasmania (Villalba et al. 2012), con aumento en las tendencias del crecimiento radial de $N$. betuloides en las últimas décadas. Los bosques de Nueva Zelandia y Tasmania muestran también un incremento en sus crecimientos, teniendo correlaciones positivas con la temperatura. En Nueva Zelandia esto estaría dado por el debilitamiento de los vientos del oeste y el gran número de masa cálidas subtropicales que han aumentado su influencia en esta región en las últimas décadas, mientras que en Tasmania la causa sería el calentamiento registrado en esta región (Villalba et al. 2012). Los bosques de $N$. betuloides del sector oeste de la Cordillera de Darwin están sometidos a condiciones oceánicas y particularmente a condiciones ambientales frías y húmedas, con influencia notoria de los frentes polares, similares a las encontradas en las vertientes occidentales de Nueva Zelandia, y Tasmania, lo que explicaría el patrón similar en el crecimiento de sus bosques.

Los dos patrones de crecimiento para los bosques de $N$. betuloides recién descritos tienen además una expresión en la composición y fisionomía de estos bosques. Es así como en los sectores en donde el patrón de crecimiento dominante es el descrito por el CP-1 u Oriental, $N$. betuloides comparte la dominancia en el dosel con otras especies arbóreas de condiciones ambientales más mésicas tales como el Nothofagus pumilio Poepp. et Endl. Krasser (lenga). Por el contrario, en los ambientes del extremo occidental de la Cordillera de Darwin, con condiciones de humedad muy altas y bajas temperaturas, y donde el patrón de crecimiento característico es el descrito por el CP-2, el dosel continuo de las especies arbóreas está totalmente dominada por $N$. betuloides sin contrapeso, conformando el muy extendido bosque perennifolio de Magallanes (Pisano 1977).

El análisis de las frecuencias dominantes en los patrones de crecimiento arbóreo de $N$. betuloides realizado en este trabajo reafirma la diferencia entre los dos principales modos de variación, CP-1 y CP-2. En el caso de CP-1, con mayor influencia de los bosques del sector oriental de la Cordillera de Darwin, las frecuencias dominantes corresponden a los períodos de dos a cuatro años. Sin embargo, el análisis de cross-wavelet permitió constatar que la estabilidad y significancia de esta señal es muy acotada en el tiempo, reduciéndose a solo dos décadas (1950-1970). En cambio, para el caso de CP-2 con frecuencias dominantes para períodos en el mismo rango de años (2,5 años), la persistencia de la señal es mucho más estable en el tiempo. La otra característica destacable es que tanto CP-1 y $\mathrm{CP}-2$ muestran una señal marginalmente significativa para el período de 16 años, la que es estable a través de todo el rango de años analizados. Al comparar mediante el análisis de cross-wavelet las frecuencias dominantes de CP-1 y CP-2, con las frecuencias dominantes descritas para el índice de oscilación Antártica AAO-NCEP, se encuentran zonas de fuerte señal espectral común en especial para la comparación CP-1 y AAO-NCEP calculado para el mes de diciembre. Esto viene a reforzar los resultados encontrados en este trabajo utilizando análisis de correlación entre el crecimiento arbóreo y variable climáticas tales como las temperaturas estivales y el patrón de circulación predominante AAO.

La relación entre la Oscilación Antártica (AAO) y los patrones de crecimiento de los bosques de $N$. betuloides descritos en este trabajo reitera lo publicado para el patrón Oriental por Villalba et al. (2012), y agrega una relación negativa significativa para el índice AAO reconstruido a partir de las anomalías de deuterio de un testigo de hielo antártico (Abram et al. 2014). Que se constaten estas relaciones entre el crecimiento arbóreo de los bosques de $N$. betuloides de la Cordillera de Darwin y los modos de circulación de la AAO contribuye a valorar estas cronologías del crecimiento arbóreo como un proxy ambiental de relevancia hemisférica.

\section{CONCLUSIONES}

A través de este estudio se logra determinar cuáles son las variables climáticas que explican un alto porcentaje de la varianza de los patrones de crecimiento de los bosques de $N$. betuloides en Cordillera de Darwin; a saber, la temperatura del aire y la precipitación durante los meses estivales, variables fuertemente asociadas a cambios en el índice de Oscilación Antártico. Para lograr este propósito central del estudio se consiguió exitosamente un primer objetivo específico con el desarrollo de nuevas cronologías de ancho de anillos a partir de muestras colectadas en condiciones ambientales que aseguran la sensibilidad del crecimiento arbóreo a las fluctuaciones climáticas. El desarrollo de las nuevas cronologías de ancho de anillos de $N$. betuloides para la Cordillera de Darwin aquí informadas contribuye a la determinación y conocimiento de la variabilidad en los patrones de crecimiento arbóreo de esta especie. Por otro lado, la determinación de dos patrones independientes de crecimiento arbóreo, CP1 y CP2, con correlaciones significativas con temperatura, variaciones isotópicas de testigo de hielo antártico y el índice AAO, da cumplimiento al segundo objetivo específico de este estudio, otorgando una mayor capacidad explicativa a la red regional de cronologías de $N$. betuloides para el extremo austral de América del Sur.

La sensibilidad a la variabilidad climática local, regional y hemisférica que muestra el crecimiento arbóreo de los bosques de la especie $N$. betuloides de la Cordillera de Darwin, demuestra que las series de ancho de anillos de esta especie pueden ser utilizadas para estimar las fluctua- 
ciones ambientales de los últimos siglos en esta región, y actuar como un sustituto de los inexistentes o muy incompletos registros climáticos instrumentales. De esta forma se confirma la hipótesis puesta a prueba al establecer relaciones significativas entre el crecimiento arbóreo de $N$. betuloides y las variables climáticas analizadas.

\section{AGRADECIMIENTOS}

Esta investigación fue financiada por el proyecto Fondecyt Regular 1030381 "Recent, centennial and multi-millenial glacier fluctuations in Cordillera de Darwin, Tierra del Fuego Island, southern Chile”. Se agradece el apoyo en el trabajo de terreno de María Pía Guzmán, Inés Dussaillant, Emilia Fercovic, Mauricio Fuentes y Hans Linderholm. Del mismo modo agradecemos las sugerencias al análisis de los datos por parte de Osvaldo Vidal y Álvaro González-Reyes. Se agradece además los comentarios de dos revisores anónimos que permitieron mejorar significativamente este trabajo.

\section{REFERENCIAS}

Abram NJ, R Mulvaney, C Arrowsmith. 2011. Environmental signals in a highly resolved ice core from James Ross Island, Antarctica. Journal of Geophysical Research 116: D20116, doi:10.1029/2011JD016147.

Abram NJ, R Mulvaney, EW Wolf, J Triest, S Kipfstuhl, LD Trusel, F Vimeux, L Fleet, C Arrowsmith. 2013. Acceleration of snow melt in an Antarctic Peninsula ice core during the twentieth century. Nature Geoscience 6: 404-411.

Abram NJ, R Mulvaney, F Vimeux, SJ Phipps, J Turner, MH England. 2014. Evolution of the Southern Annular Mode during the past millennium. Nature Climate Change 4: 564-569.

Briffa KR. 1995. Interpreting high-resolution proxy climate data: the example of Dendroclimatology. In Von Storch H, A Navarra eds. Analysis of climate variability, applications of statistical techniques. Berlin, Alemania. Springer. p. 77-93.

Bunn AG. 2008. A dendrochronology program library in $\mathrm{R}$ (dplR). Dendrochronologia 26: 115-124.

Carrasco JF, G Casassa, A Rivera. 2002. Meteorological and climatological. In Casassa G, FV Sepúlveda, RM Sinclair eds. The Patagonian Icefields: a unique natural laboratory for environmental and climate. United States of America, New York. Kluwer Academic/Plenum Publisher. p. 29-41.

Cook ER, KR Briffa, PD Jones. 1994. Spatial Regression Methods in Dendroclimatology: A review and comparison of two techniques. International Journal of Climatology
14: $379-402$

Fritts HC. 1976. Tree-rings and climate. Arizona, USA. Laboratory of Tree-Ring Research. University of Arizona. 567 p.

Garreaud RD, M Vuille, R Compagnucci, J Marengo. 2009. Present-day South American climate. Palaeogeography, Palaeoclimatology, Palaeoecology 281(3-4): 180-195.

Gillett NP, TD Kelly, PD Jones. 2006. Regional climate impacts of the Southern Annular Mode. Geophysiscal Research Letters 33: L23704.

Gouhier T, A Grinsted, V Simko. 2016. Conduct Univariate and Bivariate Wavelet Analyses: Package 'biwavelet'. Disponible en http://github.com/tgouhier/biwavelet.

Holmes RL. 1992. Dendrochronology Program Library, Instruction and Program Manual (January 1992 update). Tucson: Laboratory of Tree-Ring Research, University of Arizona.

Lara A, R Villalba, R Urrutia. 2008. A 400-year tree-ring record of the Puelo River summer-fall streaflow in the Valdivian rainforest eco-region, Chile. Climatic Change 86: 331-356.

Llancabure JJ. 2011. Relaciones entre el crecimiento de Nothofagus betuloides y el clima local y de gran escala en bosques subantárticos de la Isla Navarino. Tesis Ingeniero Forestal. Valdivia, Chile. Facultad de Ciencias Forestales y Recursos Naturales, Universidad Austral de Chile. 29 p.

Pisano E. 1977. Fitogeografía de Fuego-Patagonia Chilena. I: Comunidades vegetales entre las latitudes $52^{\circ}$ y $56^{\circ} \mathrm{S}$. Anales del Instituto de la Patagonia 8: 121-250.

Quintana JM, P Aceituno. 2012. Changes in the rainfall regime along the extratropical west coast of South America (Chile): 30-43ㅇ. Atmósfera 25(1): 1-22.

Stokes MA, TL Smiley. 1968. An introduction to tree-ring dating. Chicago, Illinois: University of Chicago Press. 73 p.

Thompson D, J Wallace. 2000. Annular Modes in the Extratropical Circulation. Part I: Month-to-Month Variability. Journal of Climate 13(694): 1000-1016.

Villalba R, A Lara, J Boninsegna, MH Masiokas, S Delgado, JC Aravena, F Roig, A Schmelter, A Wolodarsky, A Ripalta. 2003. Large-Scale tempertature changes across the Southern Andes: 20th-century variations in the context of past 400 years. Climate Change 59: 177-232.

Villalba R, A Lara, MH Masiokas, R Urrutia, BH Luckman, GJ Marshall, IA Mundo, DA Christie, E. R Cook, R Neukom, K Allen, P Fenwick, JA Boninsegna, AM Srur, MS Morales, D Araneo, JG Palmer, E Cuq, JC Aravena, A Holz, C LeQuesne. 2012. Unusual Southern Hemisphere tree growth patterns induced by changes in the Southern Annular Mode. Nature Geoscience 5: 793-798.

Wei T. 2013. Visualization of a correlation matrix: Package 'corrplot'. Disponible en https://github.com/taiyun/corrplot.

Zang C, F Biondi. 2013. Dendroclimatic calibration in R: The bootRes package for response and correlation function analysis. Dendrochronologia 31: 68-74. 